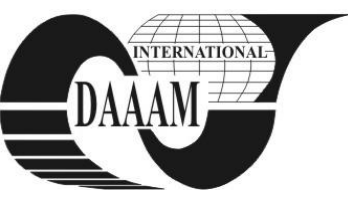

\title{
HAMMERSTEIN AND WIENER MODELS IN MODELING OF NONLINEAR PROCESS
}

\section{BABIK, Z[denek] \& DOSTAL, P[etr]}

\begin{abstract}
The application of Hammerstein and Wiener models in simulation of real nonlinear systems is described in this paper. Equations which are used for design of nonlinear model are presented too. This method has been tested on real servospeed mechanism AMIRA DR300.

Key words: Hammerstein and Wiener models, static nonlinearity, nonlinear system, servo-speed mechanism AMIRA DR300
\end{abstract}

\section{INTRODUCTION}

The nonlinear-system theory is a field of general system theory which is examined much fewer than classical linear theory. But in the practice, the most of real processes have a nonlinear behavior and their linearization is often possible only in a near neighborhood of operating points. The simulation of such processes by using of classical linear models cannot be successful.

One solution of this problem is presented in this paper. It is based on the fact that many real processes can be factorable they are consisting from nonlinear static parts and linear dynamic parts. On the bases of their mutual position Hammerstein or Wiener model can be distinguished (Nelles, 2001; Bányász at al., 2002).

\section{HAMMERSTEIN AND WIENER MODELS}

In Fig. 1 and Fig. 2 we can see the basic Hammerstein and Wiener models.

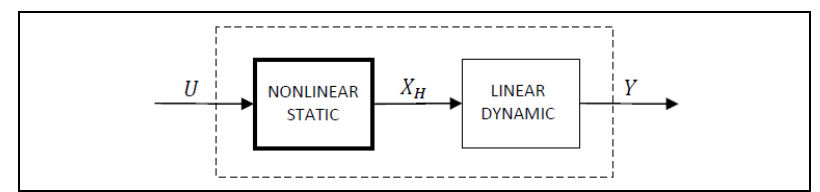

Fig. 1. Hammerstein model of nonlinear system

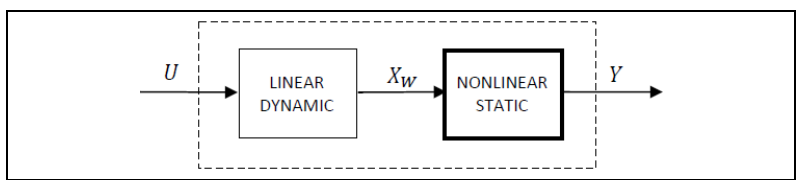

Fig. 2. Wiener model of nonlinear system

The basic Hammerstein model $\boldsymbol{N}_{\boldsymbol{H}}$ is a cascade structure of the nonlinear static block and the linear dynamic block which can be described by next formulas:

$$
\begin{gathered}
X_{H}(k)=f_{H}(U(k)) \\
Y(k)=b_{0} X_{H}(k)+b_{1} X_{H}(k-1)+\cdots+b_{m} X_{H}(k-m) \\
-a_{1} Y(k-1)-\cdots-a_{n} Y(k-n)
\end{gathered}
$$

The basic Wiener model $\boldsymbol{N}_{W}$ is a cascade structure of the linear dynamic block and the nonlinear static block which can be described by next formulas:

$$
\begin{aligned}
X_{W}(k)=b_{0} U(k)+ & b_{1} U(k-1)+\cdots+b_{m} U(k-m) \\
& -a_{1} X_{W}(k-1)-\cdots-a_{n} X_{W}(k-n)
\end{aligned}
$$

$$
Y(k)=f_{W}\left(X_{W}(k)\right)
$$

The H-models can describe many different processes, especially if their main nonlinear behavior is caused by actuators (dead zone, saturation, etc.)

The W-models are appropriate for systems, whose outputs are measured by sensors with nonlinear characteristic, and for controller design.

\section{HAMMERSTEIN PROCESS MODEL}

The equation (1) was transformed into the transfer function form. Then the Hammerstein model can be written like:

$$
\begin{aligned}
G_{H}\left(z^{-1}\right)=\frac{Y\left(z^{-1}\right)}{X_{H}\left(z^{-1}\right)} & =\frac{b_{1} z^{-1}+\cdots+b_{m} z^{-m}}{1+a_{1} z^{-1}+\cdots+a_{n} z^{-n}} \\
X_{H}(k) & =f_{H}(U(k))
\end{aligned}
$$

where: $G_{H}\left(z^{-1}\right)$ is the dynamic linear part and $f_{H}$ is the static nonlinear part.

The output value $Y(k)$ can be calculated form equation:

$$
\begin{gathered}
Y(k)=b_{1} f_{H}(U(k))+\cdots+b_{m} f_{H}(U(k-m))-a_{1} Y(k \\
-1)+\cdots+a_{n} Y(k-n)
\end{gathered}
$$

The input value $U(\cdot)$ will be considered like:

$$
U(k-m)=\cdots=U(k-1)=U(k)
$$

where: $m$ is a degree of numerator of $G_{H}\left(z^{-1}\right)$. Input signal so can be considered stable and termed $U^{S}(\cdot)$. Assume that some function $f_{\text {stat }}$ exist there, which assigns every value $U^{s}(k)$ to the value $Y^{s}(k)$ such that:

$$
Y^{s}(k)=f_{\text {stat }}\left(U^{s}(k)\right)
$$

The $f_{\text {stat }}$ is an approximation of a static characteristic of modeled system. Now we can write $f_{\text {stat }}=f_{H}$ and for output value is valid:

$$
\begin{array}{r}
Y(k)=b_{1} f_{\text {stat }}\left(U^{S}(k)\right)+\cdots+b_{m} f_{\text {stat }}\left(U^{S}(k-m)\right) \\
-a_{1} Y(k-1)+\cdots+a_{n} Y(k-n)
\end{array}
$$

From equation (9) and (10) and the transform of the result is valid:

$$
G_{H}\left(z^{-1}\right)=\frac{Y\left(z^{-1}\right)}{Y_{S}\left(z^{-1}\right)}=\frac{b_{1} z^{-1}+\cdots+b_{m} z^{-m}}{1+a_{1} z^{-1}+\cdots+a_{n} z^{-n}}
$$

\section{NONLINEAR STATIC CHARACTERISTIC OF SYSTEM AMIRA DR300}

The static characteristic of AMIRA DR300 was measured by using a multilevel measuring signal: 


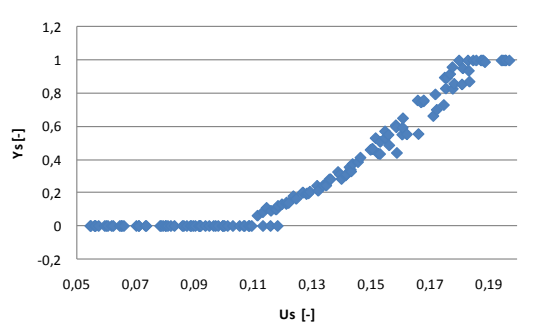

Us [-]

Fig. 3. The static characteristic of servo-speed mechanism AMIRA DR300

Now the static characteristic will be transformed to new grid system for facilitation of the approximation:

$$
X_{S}=U_{s}-U_{S}^{Y^{S}>0}=U_{s}-0.1096
$$

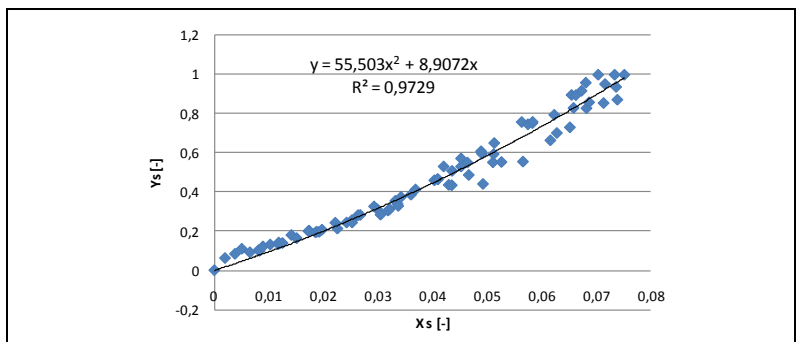

Fig. 4. The normed static characteristic of AMIRA DR300

The approximation of normed static characteristic of AMIRA DR300 will be expressed in the form:

$$
Y_{S}=55,503 X_{S}^{2}+8,9072 X_{S}
$$

The approximation of static characteristic of AMIRA DR300 will be expressed from equation (12) and (13):

$$
\begin{gathered}
U_{s} \in\left(\begin{array}{ll}
0 & 0.1096
\end{array}\right): \quad Y_{s}=0 \\
U_{s} \in\langle 0.1096 \quad 0.1848\rangle: Y_{s} \\
=55,503 U_{s}^{2}-3,2591 U_{s}-0,3095 \\
U_{s}>0.1848: \quad Y_{s}=0.9991
\end{gathered}
$$

\section{IDENTIFICATION OF DYMAMIC PART OF SYSTEM AMIRA DR300}

The transfer function of dynamic part of AMIRA DR300 will be anticipated in the form:

$$
G_{H}\left(z^{-1}\right)=\frac{Y\left(z^{-1}\right)}{Y_{S}\left(z^{-1}\right)}=\frac{b_{1} z^{-1}+b_{2} z^{-2}}{1+a_{1} z^{-1}+a_{2} z^{-2}}
$$

The transfer function parameters will be identified by the continuous minimal squares identification method. The description of this method can be founded for example in (Corriou 2004), (Bobál 2006). Only the results will be stated in this paper.

$$
\begin{aligned}
& G_{H}\left(z^{-1}\right)=\frac{Y\left(z^{-1}\right)}{Y_{s}\left(z^{-1}\right)}=\frac{0,2615 z^{-1}-0,02339 z^{-2}}{1-0,92056 z^{-1}+0,107498 z^{-2}} \\
& \quad \text { for } T_{0}=0.5 s
\end{aligned}
$$

\section{LINEAR MODEL OF SYSTEM AMIRA DR300}

The transfer function of linear model of AMIRA DR300 will be anticipated in form:

$$
G\left(z^{-1}\right)=\frac{Y\left(z^{-1}\right)}{U\left(z^{-1}\right)}=\frac{b_{1} z^{-1}+b_{2} z^{-2}}{1+a_{1} z^{-1}+a_{2} z^{-2}}
$$

The transfer function parameters will be identified by the continuous minimal squares identification method.

The linear model of AMIRA DR300:

$$
\begin{aligned}
G\left(z^{-1}\right) & =\frac{Y\left(z^{-1}\right)}{U\left(z^{-1}\right)}=\frac{3,4746 z^{-1}-3,4007 z^{-2}}{1-1,8068 z^{-1}+0,8277 z^{-2}} \\
\text { for } T_{0} & =0.5 s
\end{aligned}
$$

\section{THE COMPARE OF HAMMERSTEIN AND LINEAR MODEL OF SYSTEM AMIRA DR300}

The accuracy of Hammerstein and linear models of AMIRA DR300 will be determinate from formula:

$$
\sigma^{2}=\frac{1}{n} \sum_{I=1}^{N}\left(Y_{A M I R A}-Y_{M O D}\right)^{2}
$$

The accuracy of Hammerstein model:

$$
\sigma_{H}^{2}=0,001475
$$

The accuracy of linear model:

$$
\sigma_{L}^{2}=0,084212
$$

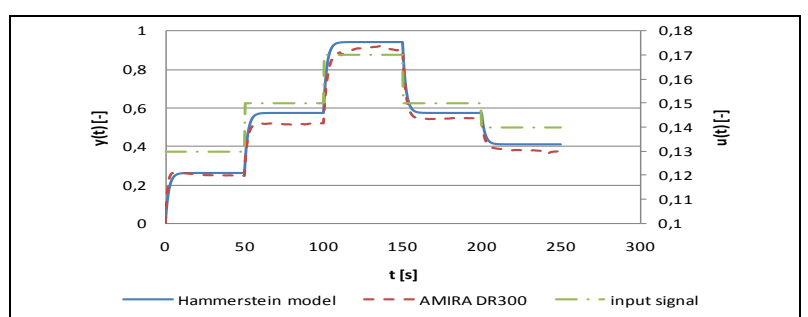

Fig. 5. Hammerstein model of AMIRA DR300

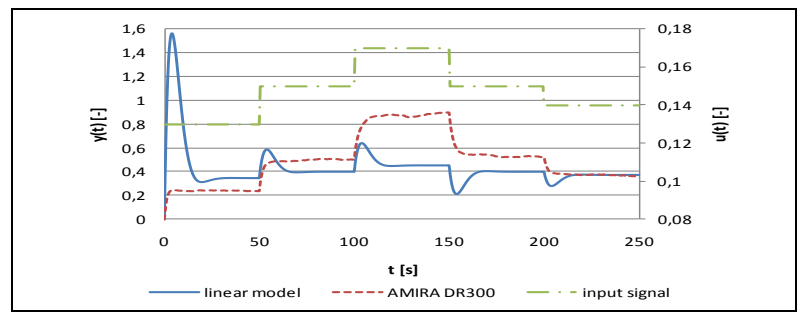

Fig. 6. Linear model of AMIRA DR300

\section{DISCUSSSION}

The following facts could be said from the measured data which are displayed in Fig. 5 and Fig. 6 and from result (22) and (23): The quality of simulation is markedly better when the Hammerstein model is used than when the linear model is used only.

On the other hand, there exist some restrictions: This method can be used only for the factorable systems and the basic version of this method is non-universal.

The universality of this method must be increased by future research. One possible way is using of methods from genetic programming field or from neural network field.

\section{ACKNOWLEDGEMENTS}

This work was partly supported by the Ministry of Education of the Czech Republic under the grant MSM 7088352101 and by TBU in Zlin under the grant IGA/55/FAI/10/D.

\section{REFERENCES}

Nelles, O. (2001). Nonlinear System Identification, 1. release, Heidelberg: Springer-Verlag, ISBN 3-540-67369-5, Berlin

Corriou, J.-P. (2004). Process control Theory and Applications, 1. release, London: Springer-Verlag, ISBN 1-85233-776-1, London

Balátě, J. (2003). Automatic control, 1. release, BEN - technical literature, ISBN 80-7300-020-2, Praque

Bobál, V. (2008). Adaptive and predictive control, 1. release, Thomas Bata University, Faculty of Applied Informatic, ISBN 80-7318-662-3, Zlín

Bányász, Cs., Keviczky L. (2002). A simple PID regulator applicable for a class of factorable nonlinear plants, In: Proceedings of the American Control Conference Anchorage, 2354-2359, ISBN 0-7803-7298-0 